\title{
A New First Arrival Pickup Algorithm Based On Information Theory for the Seismic Signals MingYue Zhai
}

\author{
School of Computer and Information Engineering \\ Guangdong University of Petrochemical Technology, Maoming, China
}

Keywords: Seismic data; First arrivals; Pickup; Information theory; Mutual information

\begin{abstract}
For the seismic data with low SNRs, the first arrival automatic picking method is very important but difficult. In the paper, we proposed a new method based on the mutual information in information theory. The mutual information between signals and noises is zeros, thus random noises have less effects on first arrivals pickup. The paper compares the principle of STA/LTA, AIC, fractal dimension of three kinds with the proposed method for seismic data first-break picking method, and at the same times, the paper presents a detailed test and verification of the simulation data, and compares first-break picking accuracy and efficiency of the three algorithms through actual data with different $\mathrm{S} / \mathrm{N}$ ratios. The results show that for the data with high $\mathrm{S} / \mathrm{N}$ ratio, first break picking accuracy of these four methods is relatively high. When SNR decreases, first-arrival time that the proposed method picks has higher precision and good noise immunity. However, mutual information based method has lower efficiency and is limited by algorithm principle. it is difficult to separately pick first breaks for fractal dimension and AIC method. So it is a very good method to identify seismic events and determine preliminarily the time range of first breaks by the proposed method.
\end{abstract}

\section{Introduction}

In seismic exploration, first-break picking is the task of determining, given a set of seismic traces, the on sets of the first signal arrivals as accurately as possible. In general, these arrivals are associated with the energy of refracted waves at the base of the weathering layer or to the direct wave that travels directly from the source to the receiver.

The accurate determination of the first arrivals onset first-break times is needed for calculating the static corrections, a fundamental stage of seismic data processing. Clearly, the effectiveness of reflection and refraction-based methods of static corrections depends on the picking-process reliability. At the same times, applications such as near-surface tomographic static corrections tomographic statics require rapid automated detection of the signal first.

Generally, first-break quality is related to the near-surface structure, source type, and signal-to-noise ratio $\mathrm{S} / \mathrm{N}$ conditions. As a consequence, the automated picking of first breaks can be a very difficult task if data area acquired in complex near-surfaces scenarios or if the $\mathrm{S} / \mathrm{N}$ is low. Moreover, if the source wavelet is zero-phase as when vibroseis sources are used, the sweep correlation often produces side-lobes that arrive before the first break, thus making the picking process even more difficult.

First arrival pickup of seismic waves, so far, has had a lot of methods. According to the criterion, first-arrival picking algorithms can be divided into several types of methods, including the coherence method, cross-correlation method, neural network method and fractal dimension methods. For the coherence and neural network methods, some kinds of patterns are assumed for picking the first arrivals. Therefore, pattern recognition of this type is effective if a simple earth model exists to model the earth structure. However, this simple earth-model rarely matches the near-surface conditions when studied with the detail required of most modern surveys. The advantages of the cross-correlation methods are that the algorithm is based on trace-by-trace evaluation of the first-arrival times and are considered to be most appropriate for the near-surface surveys.

In the paper, we proposed a robust method of first-break picking for data sets with high noise 
levels through the use of the information theory on seismic records. Using synthetic shot records with various noise levels, we showed that the performance of this proposed method enhances first arrivals, which helps in picking them. This was particularly true when the noise level was high where picking on raw amplitudes completely fails. The method can be used to guide better the subsequent careful picking of first arrivals and requires one forward. In contrast to methods based on trace-by-trace picking that often fail to pick some traces, the proposed method automatically interpolates missing picks.

\section{Basics of Information Theory}

The average amount of information gained from a given discrete space $X$ is the entropy $Y$,

$$
H(X)=-\sum_{i} P\left(x_{i}\right) \log P\left(x_{i}\right)
$$

If the $\log$ is taken to the base two, $H$ is in units of bits. In information theory, the definition of entropy of information is as follows. There is a given discrete probability space, and such space can express sources of information and the random variable $I$. The entropy of information is the mathematical expectation of $I$ and there unit is bit/symbol.

$$
H(X)=E[I(x)]=-\sum_{i} p\left(x_{i}\right) \log p\left(x_{i}\right)
$$

The conventional mutual information has been defined as:

$$
I(X, Y)=_{X, Y} f_{X Y}(x, y) \ln \frac{f_{X Y}(x, y)}{f_{X}(x) f_{Y}(y)} d x d y
$$

where, $f_{X Y}(x, y)$ is the joint probability density function (PDF), and $f_{X}(x)$ and $f_{Y}(y)$ are the marginal PDFs of variables $X$ and $Y$, respectively.

Mutual information can also be equivalently expressed as:

$$
\begin{gathered}
I(X ; Y)=H(Y)-H(Y / X) \\
I(X ; Y)=H(X)+H(Y)-H(X Y)
\end{gathered}
$$

Actually, the mutual information is very difficult to calculate since the probabilities of variables $X$ and $Y$ are quite difficult to get. In the paper, we propose a new method to calculate the mutual information based on the recursive idea. In principle, the mutual information is a kind of measuring how dependent the variable of $X$ are on the variable of $Y$.

By making the assignment, $[s, q]=[x(t), x(t+T)]$, we can consider a general system $(S, Q)$, then the uncertainty of measurement of $q$, given $(S, Q)$, is

$$
H\left(Q \mid s_{i}\right)=-\sum_{j} P_{q \mid s}\left(q_{j} \mid s_{i}\right) \log P_{q \mid s}\left(q_{j} \mid s_{i}\right)
$$

where $P_{q \mid s}\left(q_{j} \mid s_{i}\right)$ is a probability, and such a probability can measure the variable of $q$. And such a measurement can produce $q_{j}$, given that the values of $s$, is $s_{i}$. At the same time, the average uncertainty in a measurement of $x$ at the time $t+T$ can be shown given that $x$ has been measured at time $t$,

$$
H(Q \mid S)=\sum_{i} P_{s}\left(s_{i}\right) H\left(Q \mid s_{i}\right)=H(S, Q)-H(S)
$$

where,

$$
H(S, Q)=-\sum_{i, j} P_{q, s}\left(q_{j}, s_{i}\right) \log P_{q, s}\left(q_{j}, s_{i}\right)
$$


$H(Q \mid S)$ is the uncertainty of $q$ with the condition of $s . H(Q)$ is the uncertainty of $q$, and So the amount that a measurement of $s$ reduces the uncertainty of $q$ is according to the equation (4)

$$
I(Q, S)=H(Q)-H(Q \mid S)
$$

Mutual information is very important, because mutual information is not a function of the variables $s$ and $q$. It is related with the joint probability distribution $P_{s q}$. If $s$ and $q$ are similar under the condition of the noise, then $(S, Q)$ can define the measurements' relative accuracy, i.e., one measurement can give how much information in a second.

If $S$ is an image of $Q$, then the estimated joint distribution can be obtained by the delay phase portrait of $P_{s q}$ and $I$ is a variable computed by the portrait and such a variable can estimate the redundancy of the second axis.

\section{New Method for First Arrivals}

We use the software "SmartSignalProcessing" to calculate the mutual information of the arbitrary $A$ and $B$ in two channels on seismic record, and such a software was developed by Prof. Ming-Yue ZHAI at North China Electric Power University, and steps are as follows:

(1): Firstly, we reduce the seismic wave with length of $L$ on channel $A$ to: $\{x(n)\}_{n=0,1, \ldots+}$, where the length of sequences is $L$. Then equal parts are divided from the sequence $x$, whose length is $M$. .

(2): Secondly, with the sliding window, the mutual information is calculated one time. With such a method, we can obtain the mutual information $I(d)$.

(3): For $I(d), d=0,1, \ldots$, we get the maximum $I\left(D_{\max }\right)$. The corresponding time slot is the first arrival time.

In the above method, mutual information of the seismic channel $A$ and channel $B$ can be computed. Due to the complexity of mutual information calculation, we improve such a method.

By the maximum of the seismic records' mutual information, we can obtain maximum of the mutual information. The first arrival times of seismic waves are the corresponding times. The proposed method is with better accuracy than the other algorithms, such as the ratio of energy method. However, it suffer from the extensive computation load because it should calculate from the first channel to the last channel. But the advantage is that mutual information is accurate and robust,because random noises are uncorrelated with signals of interesting and thus the mutual information is zero for such case.

\section{Applications to Simulated Signals}

In the section, we applied the proposed method to a seismic profile, which is generated by the time-delayed line models(TDL). The wavelet used in TDL model is Ricker wavelet, with center frequency $10 \mathrm{~Hz}$. The simulated data without noise is illustratedin Fig.1, and the noisy version of the simulated data is plotted in Fig.2. As an example, Fig.3 illustrated the results of the proposed method. From the simulations, we can see that the proposed method can obtain teh precise first arrivals even under very low SNR.

From the figures above, we can see that the proposed algorithm can pick up the first arrivals accurately under the big SNR condition. However, when the SNR is decreasing, errors of other methods, such as the ratio of energy method, are becoming larger. When the seismic records' SNRs are below than $5 d B$, the first arrivals are not very well. The method based on the mutual information can calculate the first channel's mutual information and cal also calculate the mutural information for the last channel. At the same times, it only calculates a part of mutual information. To evaluate performances of the proposed method under different SNRs, we applied it to the simulated data with different SNRs. Fig.4 shows the results. From such a figure, errors of the estimations are very low. 


\section{Conclusions}

First arrivals pickup is a very important issue in seismic data processing. The paper proposed a new method based on the mutual information. In such method, there is no any assumption for the data. Therefore, the proposed method can be applied to any seismic data. Especially, the mentioned method can work very well under very low SNRs environments, because the mutual information between data and noise is zero, and thus noises can affect arrivals pickup as less as possible.

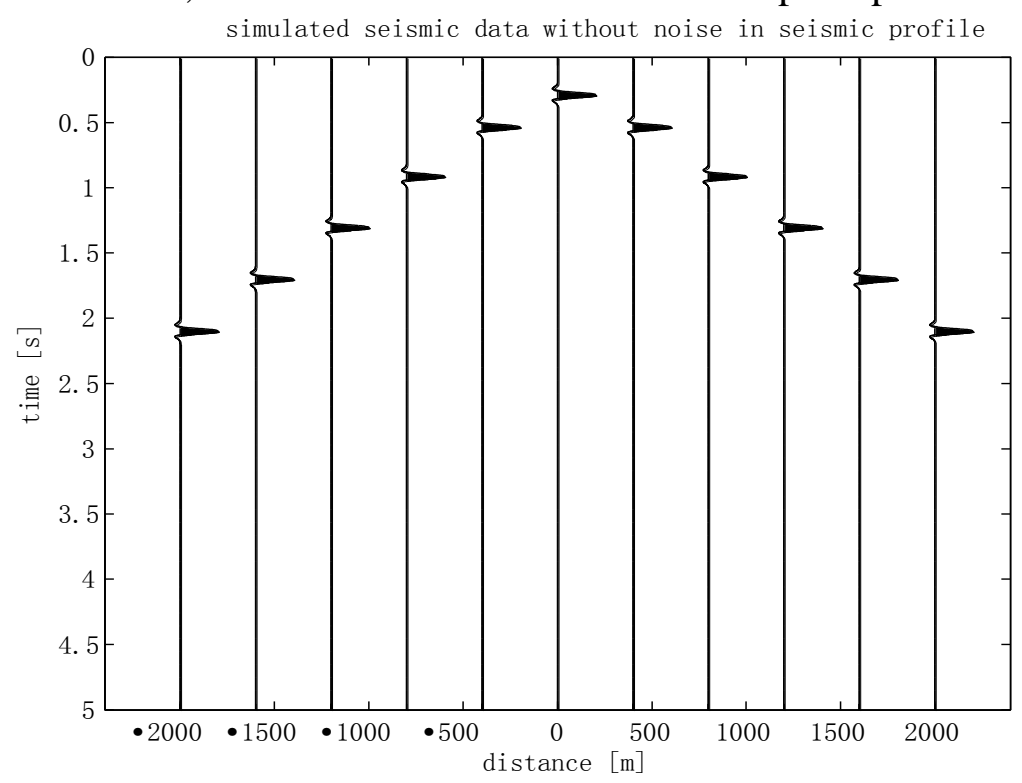

Figure 1. The simulated seismic profile with TDL model without noise

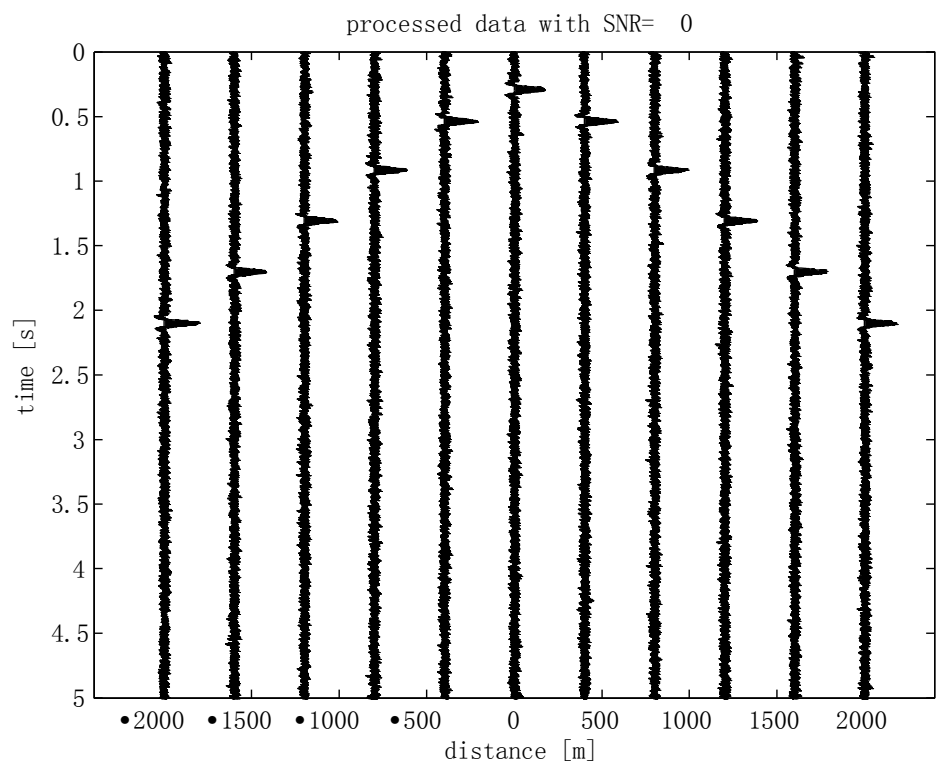

Figure 2. The simulated seismic profile with noise 

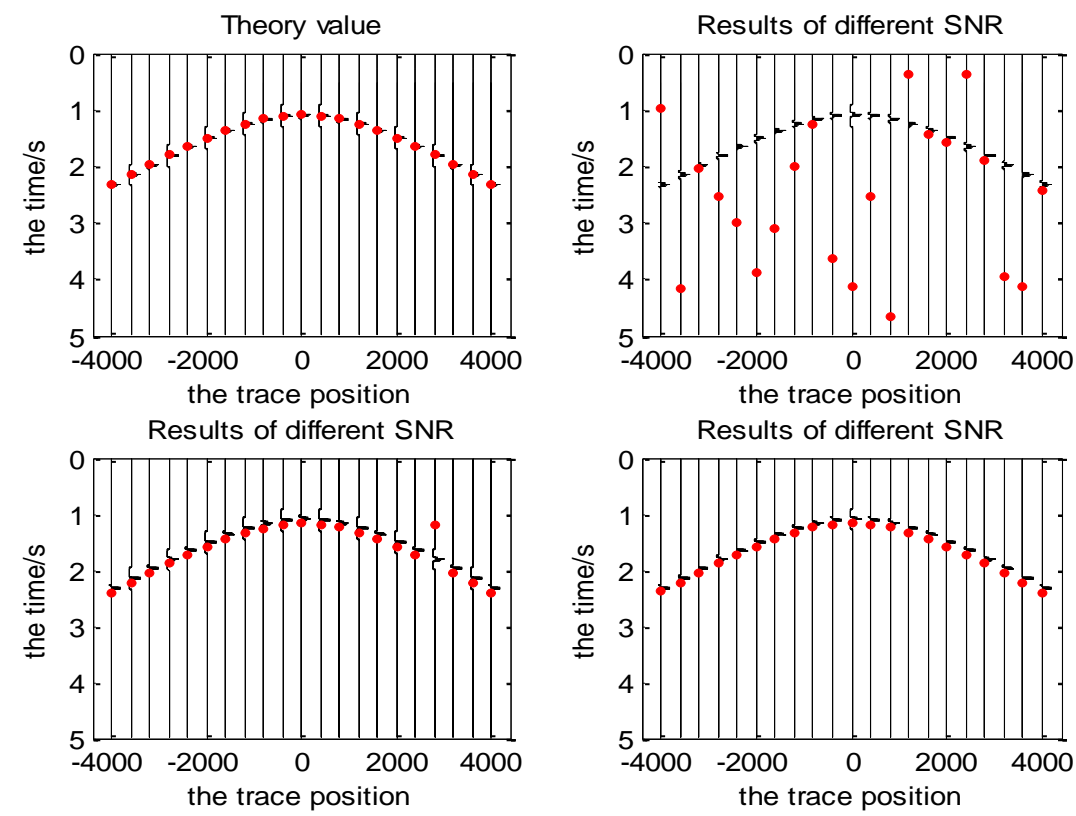

Figure 3. An example of first arrival pickup with the proposed method

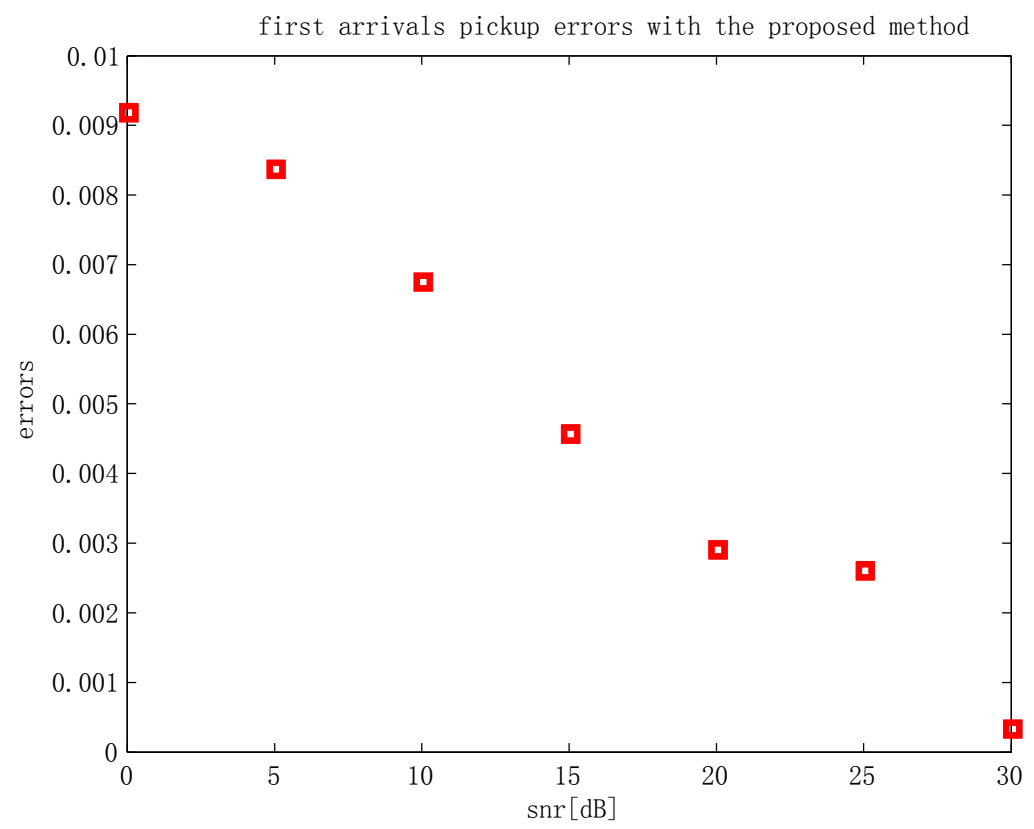

Figure 4. Errors for the first arrival pickup with the proposed method

\section{References}

[1] S. Assous, P. Elkington, S. Clark, and J. Whetton. Automated detection of planar geologic features in borehole images. GEOPHYSICS, 79(1):D11-D19, 2014.

[2] T. Keho and W. Zhu.Revisiting automatic first arrival picking for large 3D land surveys, chapter 642, pages 3198-3202. 2009.

[3] S. Li, Vladimirsky, A., and S. Fomel. First-break traveltime tomography with the double-square-root eikonal equation. GEOPHYSICS, 78(6):U89-U101, 2013.

[4] J. Sabbione and Velis D. Automatic first-breaks picking: New strategies and algorithms. GEOPHYSICS, 75(4):V67-V76, 2010.

[5] Ming-Yue Zhai. Seismic data denoising based on the fractional fourier transformation. 
JOURNAL OF APPLIED GEOPHYSICS, 109:62-70, OCT 2014.

[6] Ming-Yue Zhai, Heidi Kuzma, and James W. Rector. A new fractal-interpolation algorithm for seismic data based on iterated function systems. EPL, 92(4), NOV 2010.

[7] Ming-Yue Zhai, Juan Luis Fernandez-Martinez, and James W. Rector. A new fractal interpolation algorithm and its applications to self-affine signal reconstruction. FRACTALS-COMPLEX GEOMETRY PATTERNS AND SCALING IN NATURE AND SOCIETY, 19(3):355-365, SEP 2011. 